\title{
Transverse coronal loop oscillations seen in unprecedented detail by AIA/SDO
}

\author{
R. S. White and E. Verwichte
}

\author{
Centre for Fusion, Space and Astrophysics, Department of Physics, University of Warwick, Coventry CV4 7AL, UK \\ e-mail: r.s.white@warwick.ac.uk
}

Received 14 September 2011 / Accepted 21 October 2011

\begin{abstract}
Aims. Detailed analysis of 11 transverse coronal loop oscillations in three events observed with the Atmospheric Imaging Assembly (AIA) instrument on board the Solar Dynamics Observatory (SDO) spacecraft. Detailed analysis includes analysis of the displacement time series, intensity variations and comparing EUVI and AIA data to estimate the 3D loop geometry.

Methods. Time distance images extracted from cuts made perpendicular to the oscillations are obtained. A Gaussian plus background fitting technique is used to extract the time series which is then fitted with a damped cosine curve. Intensity variations are extracted along the time series points. EUVI/STEREO data is compared to AIA/SDO data to obtain three-dimensional models of the loop geometry.

Results. Time series analysis revealed periods between 1.7 and $10 \mathrm{~min}$ and damping times between 2.9 and 13 min. Intensity variations are reliably observed for six of the loops and a comparison between EUVI/STEREO and AIA/SDO data is performed to simulate the polarisation of the kink mode. We conclude that the intensity variations are due to variations in the line of sight column depth of a horizontally polarised transverse loop oscillation. Coronal seismology of the kink mode was applied to determine the range of the internal Alfvén speed and the magnetic field strength for each loop.
\end{abstract}

Key words. magnetohydrodynamics (MHD) - Sun: corona - Sun: oscillations

\section{Introduction}

Transverse coronal loop oscillations (TLOs) have been spatially observed since 1998 (Nakariakov et al. 1999; Aschwanden et al. 1999) and the majority of observations have been made using EUV images by the Transition Region and Coronal Explorer (TRACE). These include observations of TLOs in post-flare arcades (Verwichte et al. 2004), multiple harmonics (e.g. Verwichte et al. 2004; Van Doorsselaere et al. 2007; De Moortel \& Brady 2007), vertically polarised modes (e.g. Wang \& Solanki 2004; Mrozek 2011), large amplitude TLOs (Verwichte et al. 2010) and TLOs in cooling loops (Aschwanden \& Terradas 2008; Morton \& Erdélyi 2009). TLOs have also been reported in EUV images from other spacecraft, i.e. with the Solar Terrestrial Relations Observatory (STEREO) (Verwichte et al. 2009), SoHO (Verwichte et al. 2010) and recently with SDO (Aschwanden \& Schrijver 2011; McIntosh et al. 2011). TLOs have also been reported in spectral data (Hori et al. 2005, 2007; Tomczyk et al. 2007; Van Doorsselaere et al. 2008b).

In terms of the magnetohydrodynamic (MHD) wave theory, transverse loop oscillations are interpreted as the nearly incompressible fast kink (Alfvénic) mode (Edwin \& Roberts 1983; Nakariakov \& Verwichte 2005; Van Doorsselaere et al. 2008a; Goossens et al. 2009) where the fundamental mode is characterised by the displacement of the loop axis with all parts of the loop in phase (Nakariakov et al. 1999). Analysis of the kink mode has lead to the application of the technique of coronal seismology by which physical parameters of the corona such as magnetic field strengths and densities may be indirectly determined (Nakariakov \& Ofman 2001; Goossens et al. 2008). Seismology techniques may also provide information on the radial density structuring of coronal loops (Verwichte et al. 2006; Arregui et al. 2007). Since TLOs are often observed to be triggered by dynamic events such as flares information about the physical conditions within and surrounding these active regions may be investigated (Roberts 2000). An interesting feature of TLOs is their tendency to damp on short time scales, which cannot be explained by classic dissipation of the plasma inside the loops (Nakariakov et al. 1999). This has lead to much discussion on the nature of the mechanisms behind this damping (Ruderman \& Roberts 2002). Suggested mechanisms include the theories of phase mixing (Heyvaerts \& Priest 1983) and resonant absorption (Hollweg \& Yang 1988; Goossens et al. 2002) for which scaling relationships between the period and damping times have been derived (Ofman \& Aschwanden 2002; Goossens et al. 2002). In February 2010 NASA's Solar Dynamics Observatory (SDO) spacecraft was launched, which carries on board the Atmospheric Imaging Assembly (AIA) instrument (Lemen et al. 2011). AIA provides continuous observations of the full solar disk in a range of plasma temperatures with a time cadence of around 12 seconds. This allows observations of dynamics on much shorter time scales then have previously been observed by spacecraft such as TRACE and STEREO. The first detailed analysis of a transverse loop oscillation observed by AIA/SDO has been performed by Aschwanden \& Schrijver (2011). Observations of TLOs have shown variations in intensity with the same periodicity as the loop displacement (Wang \& Solanki 2004; Verwichte et al. 2009, 2010; Mrozek 2011; Aschwanden \& Schrijver 2011). They have been interpreted as due to variations in the line of sight column depth (Cooper et al. 2003; Verwichte et al. 2009), variations in density from a linearly combined fast kink and slow magnetoacoustic oscillation in a 

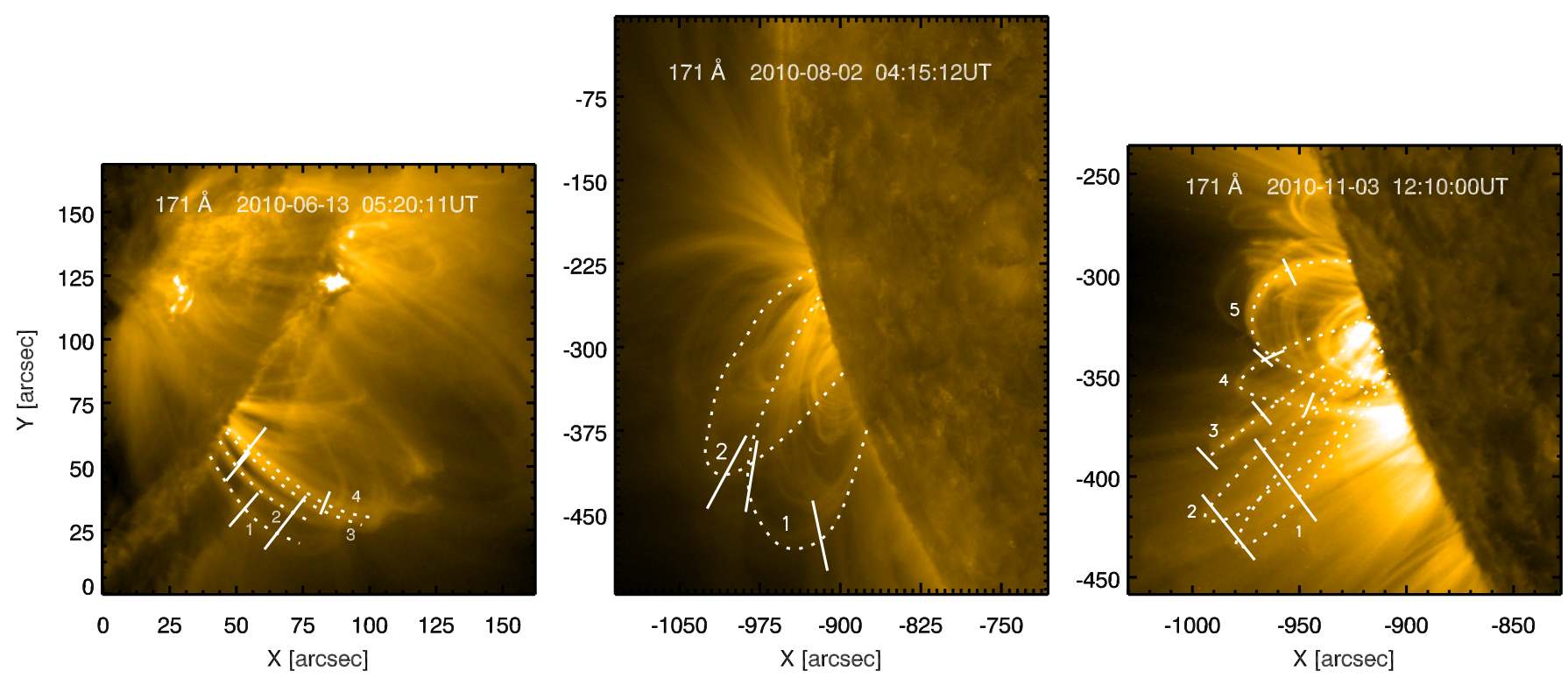

Fig. 1. AIA/SDO $171 \AA$ images of the three events indicating the loops of interest. Left: Event 1 . White tick marks indicate the regions within which cuts are taken to study the oscillations of the four loops. Center: Event 2 where two larger, fainter loops are studied. The three cuts taken to analyse the oscillations are displayed. Right: Event 3 where five loops are studied. Again white tick marks indicate the regions within which cuts are taken to study the oscillations.

finite plasma- $\beta$ loop (Terradas et al. 2011), and variations in loop length and/or cross-section in case of vertically polarised TLOs (Wang \& Solanki 2004; Verwichte et al. 2006; Aschwanden \& Schrijver 2011). Non linear coupling of a kink mode to a slow mode also generates density perturbations but which are expected to be with a different periodicity (Terradas \& Ofman 2004).

In this paper eleven coronal loops observed in three events are studied using data from AIA/SDO. This paper presents the first AIA study of multiple loops in the same active region. By studying multiple loops we investigate whether the loops in a singe active region have similar properties. We also expand the range of observed periods and damping times. In particular, the capabilities of the AIA instrument allow this range to be extended towards shorter time periods than have previously been observed. Finally, we show that intensity variations in transverse loop oscillations are a common feature. In Sect. 3 the displacement time series are presented and discussed, in Sect. 4 AIA data has been complemented with data from STEREO to estimate the 3D loop geometry, in Sect. 5 the intensity variations along the loops are investigated and in Sect. 6 seismology of the kink mode has been applied to the results. Finally, the conclusions are presented in Sect. 7.

\section{Observations}

Data sets from three time intervals from AIA/SDO are studied. AIA observes the sun in seven EUV bandpasses: Fe XVIII

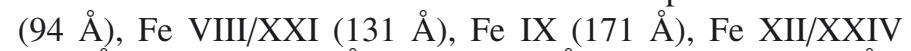
(193 $\AA)$, Fe XIV (211 $)$, He II (304 $\AA$ ) and Fe XVI (335 $\AA)$. It is able to provide continuous full disk $\left(41^{\prime} \times 41^{\prime}\right)$ images with a time cadence of approximately $12 \mathrm{~s}$, spatial resolution of $1.5^{\prime \prime}$ and a pixel size of $0.6^{\prime \prime}$. In this study the primary focus is on the $171 \AA$ bandpass which corresponds to coronal plasma temperatures of approximately $1 \mathrm{MK}$. All data sets have a time cadence of $12 \mathrm{~s}$ and are normalised by the exposure time prior to the analysis.
Images of the three events are shown in Fig. 1. Event 1 was observed between 05:20:11 and 06:19:59 UT on June 13th 2010 in active region NOAA 11079 situated on the south west limb. An M1.0 GOES class flare erupted, initially pushing the loops away from the active region and triggering oscillations which were observed to damp within $5 \mathrm{~min}$. Four loops indicated in Fig. 1 are studied. Event 2 was observed between 04:15:12 and 05:29:48 UT on August 2nd 2010 in active region NOAA 11095 situated on the south east limb. A dynamic event occurring at approximately $\left(918^{\prime \prime} \mathrm{E}, 261^{\prime \prime} \mathrm{S}\right)$ relative to the sun centre perturbed the loops and the oscillations of loops indicated in Fig. 1 are studied. These loops are much larger, have a lower contrast to the background plasma and oscillate more gently than the loops studied in the other two events. Event 3 was observed between 12:10:00 and 13:09:48 UT on November 3rd 2010 in active region NOAA 11121. This event has previously been studied by Reeves \& Golub (2011); Foullon et al. (2011); Cheng et al. (2011). Following a C4.9 GOES flare and associated CME, transverse loop oscillations are observed, which have not yet been investigated. Of these, five loops are studied which, similar to the other events, display short damping times on the order of 3 to $7 \mathrm{~min}$.

Where possible data from AIA/SDO is complemented with data from the Extreme Ultraviolet Imager (EUVI) instrument on board the STEREO spacecraft in the $171 \AA$ and $195 \AA$ bandpasses in order to obtain an estimate of the 3D loop geometry. Comparing the 3D loop geometry also allows an estimate of the loop length to be made. The EUVI instrument observes the sun in four bandpasses with a pixel size of $1.6^{\prime \prime}$ and a circular field of view of 1.7 solar radii (Howard et al. 2008).

\section{Displacement time series analysis}

Time series analysis of the transverse coronal loop oscillations is performed by examining image cuts in each of the data sets. Cuts are made across the oscillating loops parallel to the projected displacement direction of the loops. For each cut, a twodimensional time-distance image is created where the spatial 
$x$-coordinate represents the distance along the cut. The cut data has been averaged over the width of the cut, i.e. 11 pixels, to increase signal to noise. Figure 1 indicates the regions within which the oscillations are investigated for Events 1 and 3 and the location of the cuts used for the analysis of the loops in Event 2. The number of cuts made in Events 1 and 3 differ for each loop depending on how clear the oscillations are observed.

Loop features in the time-distance images are enhanced by filtering with a two-dimensional continuous wavelet transform using the Mexican hat mother wavelet function (Witkin 1983). The Mexican hat wavelet is the negative Laplacian of a Gaussian shape and enhances intensity ridges in images. It is therefore well suited to enhance the location of solar features including coronal loops (Delouille et al. 2005). The continuous Mexican hat wavelet transform is defined as

$$
\begin{aligned}
C W T(I)(\boldsymbol{b}, a) & =-\frac{1}{a^{n-2}} \int_{-\infty}^{+\infty} I(\boldsymbol{r}) \nabla^{2}\left(\mathrm{e}^{-\frac{|\boldsymbol{r}-b|^{2}}{2 a^{2}}}\right) \mathrm{d} \boldsymbol{r} \\
& =-\frac{1}{a^{n-2}} \int_{-\infty}^{+\infty} \nabla^{2} I(\boldsymbol{r}) \mathrm{e}^{-\frac{|\boldsymbol{r}-\boldsymbol{b}|^{2}}{2 a^{2}}} \mathrm{~d} \boldsymbol{r},
\end{aligned}
$$

where the value of $n$ is determined by the choice of norm (e.g. for $L_{1}$ norm, $n=2$ ). From Eq. (1) it can be seen that the Mexican hat wavelet transform represents a Gaussian blurring of the Laplacian of the image signal.

Figure 2 shows an example of one of the cuts used in the analysis and three time distance images for loop 2 from Event 1 after the application of the wavelet transform. Loop 2, indicated by the dashed line, is clearly visible in the cuts. However, loops 3 and 4 form part of a bundle of loops below loop 2 and are much more difficult to identify due to the overlapping loops and loop strands.

To extract displacement time series information a Gaussian plus second order polynomial function for the background is fitted to each time slice in the time distance images to determine the exact position of the loops. The intensity profile of a loop is well represented by a Gaussian shape (Carcedo et al. 2003). For an isolated loop with no or few interactions with other loops and a high contrast with the background this method is successful in automatically extracting the loop location. However, for many of the loops the time series had to be determined manually by eye. Figure 2 shows the automatically determined time series points plotted on the cuts for loop 2 in Event 1. To check that an extracted time series correctly matches to a loop in the SDO data set, a marker is placed on each image corresponding to the determined location of a particular loop. As the images of the event are consecutively displayed, the marker can be observed to follow a loop as it oscillates back and forth. Once the time series has been determined a 3 rd order polynomial trend is subtracted from the series and a damped cosine function of the form

$\xi(t)=\xi_{0} \exp \left(\frac{-\left(t-t_{0}\right)}{\tau}\right) \cos \left(\frac{2 \pi}{P}\left(t-t_{0}\right)-\phi\right)$

is fitted. Where $\xi$ is the amplitude, $\tau$ is the damping time, $P$ is the period, $\phi$ is the phase and $t_{0}$ is the reference time. This Levenberg-Marquardt least squares fitting was performed using the function MPFIT (Markwardt 2009) in the interactive data language (IDL). The parameter uncertainties are estimated by scaling the 1 sigma parameter errors computed from the covariance matrix by the measured chi $1 / N$ squared value, assuming that the reduced chi-squared value is one. The error in the loop location is estimated as the width of the Gaussian function fitted to each of the time slices and is approximately one $\mathrm{Mm}$. For manually determined loop locations the Gaussian width is
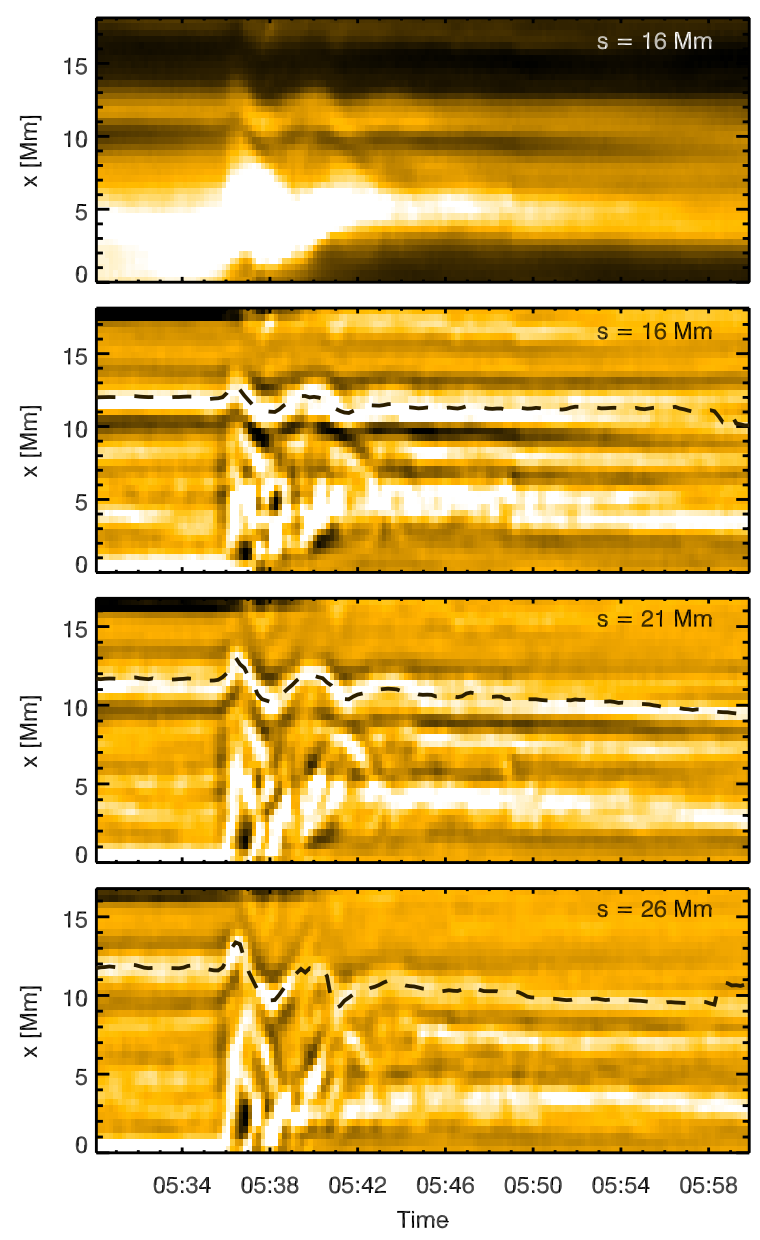

Fig. 2. Time distance images extracted from cuts taken to analyse loop 2 in Event 1. The top cut shows an example of the time distance images before a 2D mexican hat wavelet transform has been applied to enhance oscillatory features, as shown by the bottom three cuts. The automatically determined time series from the Gaussian plus background fit for loop 2 has been plotted on the wavelet enhanced cuts.

assumed to be one Mm. Figure 3 shows the displacement time series for loop 2 in Event 1 which has been normalised by the loop length, $L$, and fitted with the damped cosine curve, Eq. (2). The typical size of the error is indicated by the error bars in the figure. The loop length is determined by either assuming that the height of the loop is equal to the radius of a semi circle or by comparing the 3D loop geometry with STEREO as discussed in Sect. 4. It is expected that the error on the loop length is approximately $10 \%$ in line with previous studies (Van Doorsselaere et al. 2007). Figure 3 shows that the damped cosine function fits well to the period of the oscillation. There is a greater uncertainty in the damping time. This is to be expected because as the oscillation is damped it becomes more difficult to identify peaks and troughs due to the resolution. The time series clearly show that the loop is damped on a short time scale, within a few periods. As the distance along the loop, $s$, increases towards the apex, the displacement amplitude also increases.

Table 1 shows the oscillation parameters determined from the time series analysis of the eleven loops studied in the three events. In some cases the loop oscillations are observed to begin at slightly different times in the time distance images, either because of a slight delay in the excitation at different points along the loop or because the oscillations are not reliably identified until a later time. The amplitudes recorded are the maximum 
Table 1. Displacement oscillation parameters of the eleven loops studied, determined from the damped cosine fitting.

\begin{tabular}{cccccccccc}
\hline \hline Event & Loop & $\begin{array}{c}L \\
(\mathrm{Mm})\end{array}$ & $\begin{array}{c}\theta \\
\left({ }^{\circ}\right)\end{array}$ & $\begin{array}{c}t_{0} \\
(\mathrm{UT})\end{array}$ & $\begin{array}{c}P \\
(\mathrm{~s})\end{array}$ & $\begin{array}{c}\tau \\
(\mathrm{s})\end{array}$ & \multicolumn{1}{c}{$\begin{array}{c}\phi \\
\left({ }^{\circ}\right)\end{array}$} & $\begin{array}{c}\xi_{0} \\
(\mathrm{Mm})\end{array}$ & $\begin{array}{c}V_{\mathrm{ph}} \\
\left(\mathrm{km} \mathrm{s}^{-1}\right)\end{array}$ \\
\hline 1 & 1 & $121 \pm 2$ & $38 \pm 3$ & $05: 35: 59$ & $225 \pm 40$ & $240 \pm 50$ & $150 \pm 70$ & $3.26 \pm 0.3$ & $1080 \pm 220$ \\
& 2 & $111 \pm 6$ & $26 \pm 5$ & & $215 \pm 5$ & $293 \pm 20$ & $69 \pm 10$ & $2.76 \pm 0.2$ & $1030 \pm 110$ \\
& 3 & 132 & $\ldots$ & & $213 \pm 9$ & $251 \pm 40$ & $80 \pm 10$ & $1.92 \pm 0.1$ & $1240 \pm 140$ \\
& 4 & $113 \pm 4$ & $12 \pm 3$ & & $216 \pm 30$ & $230 \pm 20$ & $121 \pm 20$ & $4.99 \pm 0.3$ & $1050 \pm 170$ \\
\hline 2 & 1 & 396 & $\ldots$ & $04: 24: 12$ & $520 \pm 5$ & $735 \pm 50$ & $194 \pm 3$ & $1.88 \pm 0.1$ & $1520 \pm 150$ \\
& 2 & 374 & $\ldots$ & & $596 \pm 50$ & $771 \pm 340$ & $170 \pm 30$ & $3.00 \pm 0.1$ & $1260 \pm 160$ \\
\hline 3 & 1 & $279 \pm 3$ & $27 \pm 1$ & $12: 13: 36$ & $212 \pm 20$ & $298 \pm 30$ & $20 \pm 20$ & $5.97 \pm 0.3^{*}$ & $2630 \pm 360$ \\
& 2 & $240 \pm 4$ & $29 \pm 1$ & & $256 \pm 20$ & $444 \pm 110$ & $107 \pm 20$ & $7.76 \pm 0.5^{*}$ & $1880 \pm 250$ \\
& 3 & 241 & $\ldots$ & & $135 \pm 9$ & $311 \pm 90$ & $155 \pm 60$ & $3.40 \pm 0.4^{*}$ & $3570 \pm 430$ \\
& 4 & $159 \pm 6$ & $-17 \pm 1$ & & $115 \pm 2$ & $175 \pm 30$ & $80 \pm 20$ & $2.63 \pm 0.3$ & $2770 \pm 280$ \\
& 5 & 132 & $\ldots$ & $12: 14: 36$ & $103 \pm 8$ & $242 \pm 110$ & $135 \pm 30$ & $2.01 \pm 0.4$ & $2560 \pm 330$ \\
\hline
\end{tabular}

Notes. * Indicates recorded amplitudes that are at the approximate loop apex positions.

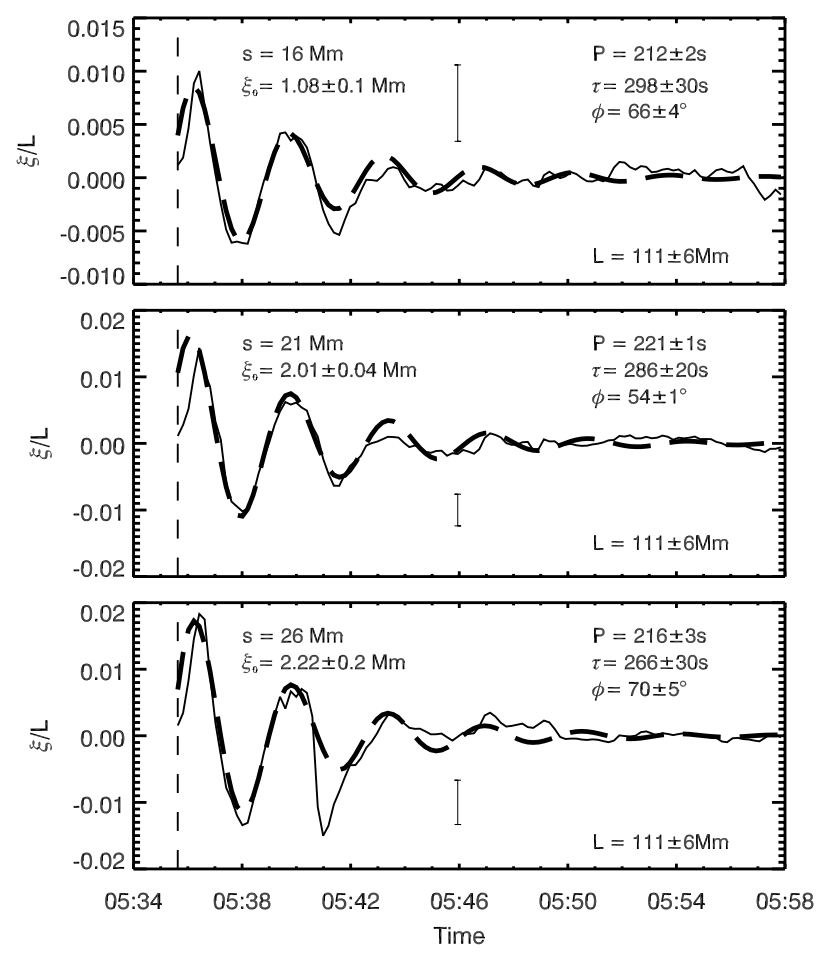

Fig. 3. Automatically determined time series (solid line) for three of the cuts taken to analyse loop 2 in Event 1 fitted with a damped cosine function (thick dashed line). Top: $s=16 \mathrm{Mm}$. Center: $s=21 \mathrm{Mm}$. Bottom: $s=26 \mathrm{Mm}$. The errors on the fitting parameters are the errors given by the fitting technique. The error bars on each plot show the typical size of the error in the loop position. The displacement amplitude has been normalised by the loop length. The vertical dashed line indicates the start of the oscillation at $t_{0}=05: 35: 59 \mathrm{UT}$.

amplitudes determined for each loop from the damped cosine fits. They do not necessarily represent the maximum amplitudes found at the apex position. The errors quoted for the periods, damping times and phases are determined by taking the standard deviation of the parameters obtained in each cut because the values from the different cuts do not lie within each others error bars (Verwichte et al. 2004; Van Doorsselaere et al. 2007). For loop 1 in Event 2 only one cut is analysed therefore the parameter errors quoted are those returned by the parameter fitting technique. Phase speeds are also calculated since $V_{\mathrm{ph}}=2 L / P$ for a fundamental of the kink mode where $L$ is the loop length. For the phase speed calculations a $10 \%$ error on $L$ was assumed. The loop lengths that have quoted errors have been determined by comparing EUVI with AIA data (see Sect. 4). In these cases the error is a measure of the precision of the method repeated several times rather than a measure of the accuracy and so a $10 \%$ error was used for the phase speed calculations. For the remaining loops the length has been determined by assuming that the height of the loop is equal to the radius of a semi circle. We now discuss each event in more detail.

\subsection{Event 1}

Three cuts are made to analyse loops 1 and 3, five cuts are made to analyse loop 2 and two cuts are made to analyse loop 4 . The periods observed are between 3.5 and 3.75 min and the damping times, which are close in value to the periods, show that these loops undergo rapid damping. Problems were encountered when trying to obtain the time series for loops 3 and 4 as these loops appear to be part of a loop bundle consisting of several overlapping loops or loop strands with slightly different periods. To identify these loops and to obtain an estimate of the loop parameters, the method described above of plotting a marker on each of the images was applied. Estimating the loop length from the height of the loop for loops 1,2 and 4 gives values of $132 \mathrm{Mm}, 126 \mathrm{Mm}$ and $132 \mathrm{Mm}$ respectively. This corresponds to percentage differences of $8 \%, 13 \%$ and $16 \%$ with the estimates from the EUVI/AIA comparison shown in Table 1. Using these lengths, the phase speeds become $1170 \pm 240 \mathrm{~km} \mathrm{~s}^{-1}, 1170 \pm$ $120 \mathrm{~km} \mathrm{~s}^{-1}$ and $1220 \pm 210 \mathrm{~km} \mathrm{~s}^{-1}$ respectively. It is expected that the lengths obtained using the EUVI/AIA comparison are more accurate than those from the height estimates because the loops are observed on the limb and the lengths are determined using information of the 3D loop geometry.

\subsection{Event 2}

Two larger loops with periods of just under $10 \mathrm{~min}$ and damping times of just under 13 min are observed. Because of the low contrast of the loops with the background and the gentle oscillations, the results are only obtained from one cut for loop 1 and two cuts for loop 2. The error on the loop length is expected to be at least $10 \%$ because the height of the loop was difficult to estimate and a comparison with EUVI data could not be performed as the loops are not observed in these images. Periods, damping times, and phases of the two loops are similar to each other. There have been few observations of loops with periods around $10 \mathrm{~min}$ but the results of Event 2 are consistent with these previous studies 
R. S. White and E. Verwichte: Transverse coronal loop oscillations seen in unprecedented detail by AIA/SDO
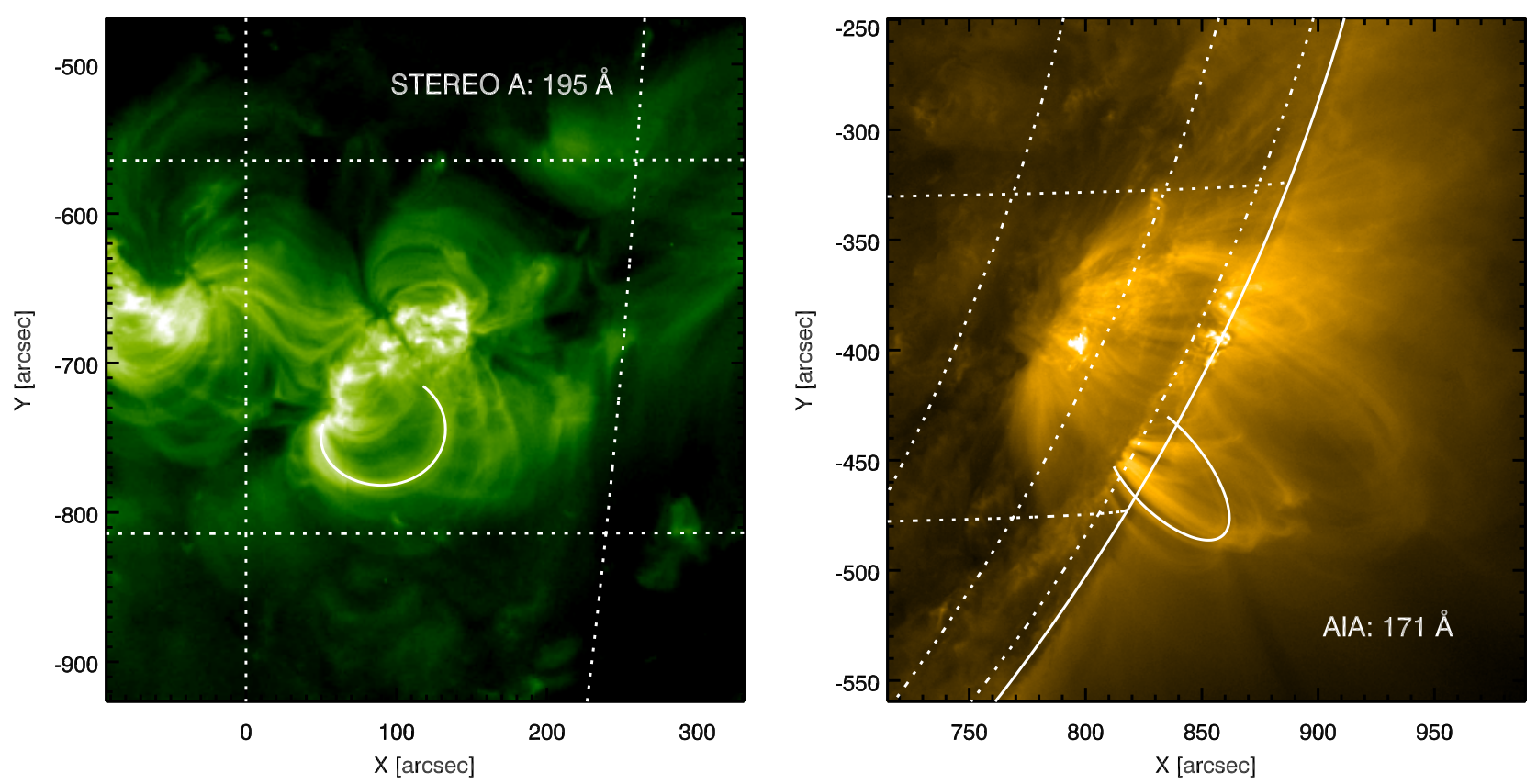

Fig. 4. Left: EUVI $195 \AA$ image from STEREO A showing loop points chosen on active region NOAA 11079, Event 1. Right: AIA/SDO 171 A image showing the projected loop coordinates chosen on the EUVI image to compare the 3D loop geometry. The inclination and azimuthal angles are $\theta=26^{\circ} \mathrm{S}$ and $\alpha=69^{\circ}$ respectively.

(Aschwanden et al. 2002; Verwichte et al. 2009). There are several loops in this event that have not been analysed because of the gentle nature of the oscillations causing oscillation amplitudes that are not large enough to be observed clearly in the time distance images.

\subsection{Event 3}

Five loops are observed and studied. Similar to the other two events strong damping is observed and the phase speeds are noted to be 2-3 times larger than the other two events. Significantly periods of less than two minutes are observed for loops 4 and 5 which is only possible to detect because of the short time cadence of AIA/SDO. All of the loops seem to be close in phase however the corrected time for loops 1 and 5 are different from the other loops because the oscillations are observed to begin slightly later. Similar to Event 1, estimates of the length for loops 1,2 and 4 from assuming the height is equal to the radius of a semi circle gives values of $248 \mathrm{Mm}, 264 \mathrm{Mm}$ and $168 \mathrm{Mm}$. This corresponds to percentage differences of $12 \%$, $10 \%$ and $6 \%$ respectively with the EUVI/AIA comparison estimates. Using these lengths, the phase speeds are calculated to be $2340 \pm 320 \mathrm{~km} \mathrm{~s}^{-1}, 2060 \pm 260 \mathrm{~km} \mathrm{~s}^{-1}$ and $2920 \pm 300 \mathrm{~km} \mathrm{~s}^{-1}$ respectively. Again it is expected that the lengths obtained using the EUVI/AIA comparison are more accurate than those from the height estimates.

\section{Comparison of EUVI/STEREO and AIA/SDO}

A comparison is made between EUVI/STEREO and AIA/SDO data to determine where possible the 3D loop geometry and to simulate the polarisation of the kink mode. We construct a three dimensional loop model from the two dimensional projected loop coordinates by assuming a planar loop, which is inclined with respect to the photospheric normal by an angle $\theta$ (Verwichte et al. 2010). By comparing the loop projections from two viewpoints (e.g. SDO and STEREO), $\theta$ can be constrained.
To compare the events in the two different instruments, chosen loop image coordinates in EUVI are converted to heliographic Cartesian and then to heliocentric Aries ecliptic coordinate systems. These coordinates are then converted back into the AIA coordinate system and displayed on an AIA image. Figure 4 shows an example of loop points chosen in an EUVI image converted and displayed on an AIA image for Event 1 for the optimal inclination angle, $\theta=26^{\circ} \mathrm{S}$. The reverse process from AIA to EUVI can also be performed however this does not always work because of the uncertainty in the footpoint locations for the events on the limb. Loop coordinates are determined for a chosen inclination angle and then compared to the loops displayed in the images to see if there is a close match. Table 1 shows the estimated loop lengths, $L$, and inclination angles, $\theta$, determined using this method. The errors on the loop lengths and inclination angles are given by the standard deviations of repeated measurements and are a measure of the precision of the method using the planar loop model. It is expected that there is an error on the loop length of at least $10 \%$. Directly comparing the loops in the EUVI and AIA images is difficult because EUVI has a worse spatial resolution than AIA and there is an uncertainty in distinguishing the loops from one another. For Event 2 it is not possible to distinguish and identify the individual loops in the EUVI image because of poor contrast against the solar disk. Therefore, this method gives here an estimate of the general loop arcade geometry rather than a detailed fit to each loop separately.

\section{Intensity variations}

Intensity variations are reliably observed in the time distance images for six of the loops, loops 2 and 3 in Event 1 and loops 1-3 and 5 in Event 3. Figure 5 shows intensity variations observed in three of the cuts for loop 2 in Event 1. The images have been saturated to clearly show the variations in intensity. The intensity at each time series point is recorded and the extracted relative intensity time series are analysed using the same procedure of subtracting a background and fitting a damped cosine 

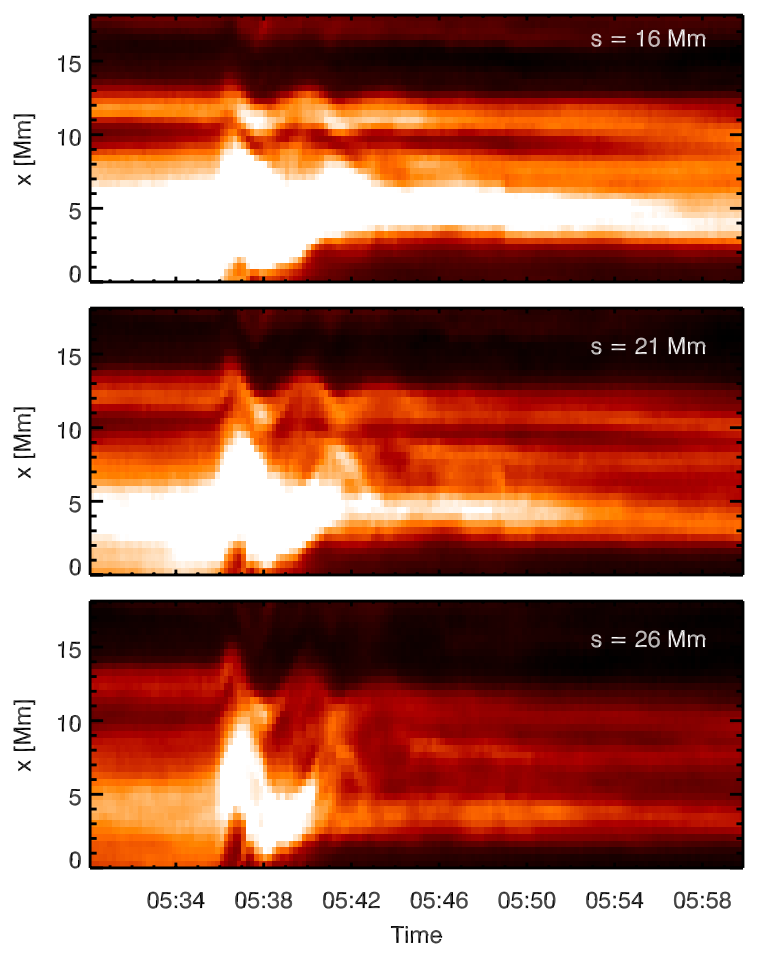

Fig. 5. Time distance images taken from three cuts used to analyse loop 2 in Event 1. The images have been saturated to highlight the intensity variations along the loop. Loop 2 is the isolated loop above the bundle of loops.

Table 2. Intensity oscillation parameters determined for each event.

\begin{tabular}{lccccc}
\hline \hline Event & Loop & $I_{\mathrm{A}}$ & $\begin{array}{c}P \\
(\mathrm{~s})\end{array}$ & $\begin{array}{c}\tau \\
(\mathrm{s})\end{array}$ & $\begin{array}{c}\phi \\
\left({ }^{\circ}\right)\end{array}$ \\
\hline 1 & 2 & $0.22 \pm 0.02$ & $215 \pm 2$ & $350 \pm 30$ & $213 \pm 10$ \\
& 3 & $0.22 \pm 0.02$ & $247 \pm 40$ & $313 \pm 20$ & $184 \pm 70$ \\
\hline 2 & 1 & $(0.06)$ & $(550)$ & $(552)$ & $(126)$ \\
& 2 & $(0.05)$ & $(612)$ & $(422)$ & $(300)$ \\
\hline 3 & 1 & $0.06 \pm 0.01$ & $306 \pm 3$ & $623 \pm 70$ & $75 \pm 50$ \\
& 2 & $0.16 \pm 0.02$ & $235 \pm 20$ & $407 \pm 160$ & $90 \pm 40$ \\
& 3 & $0.27 \pm 0.02$ & $148 \pm 40$ & $224 \pm 60$ & $114 \pm 10$ \\
& 4 & $(0.22)$ & $(130)$ & $(180)$ & $(50)$ \\
& 5 & $0.10 \pm 0.02$ & $99 \pm 2$ & $314 \pm 140$ & $94 \pm 30$ \\
\hline
\end{tabular}

Notes. The intensity amplitude $I_{\mathrm{A}}$ is the background subtracted intensity that has been normalised by the background. It is the maximum intensity given by the damped cosine fit. Intensity variations for the loops with parameters in brackets were not reliably observed in the time distance images. The period for loop 1, Event 3 is for the case $s=81 \mathrm{Mm}$.

function as discussed above for the displacement time series analysis. The extracted intensity time series does not always display clear oscillations that can be fitted with the damped cosine function. Figure 6 shows the intensity time series for loop 2 in event 1 fitted with the damped cosine function. The intensity has been normalised by the subtracted background intensity. It shows that there are significant intensity variations of between approximately $10 \%$ and $30 \%$ of the background intensity, which are in antiphase with the displacement oscillation. As the distance along the loop increases from the footpoint to the apex, the intensity amplitude increases. This suggests that the intensity amplitude scales with the displacement amplitude.

Table 2 shows the results for the intensity time series analysis of the three events. The results include the analysis of intensity
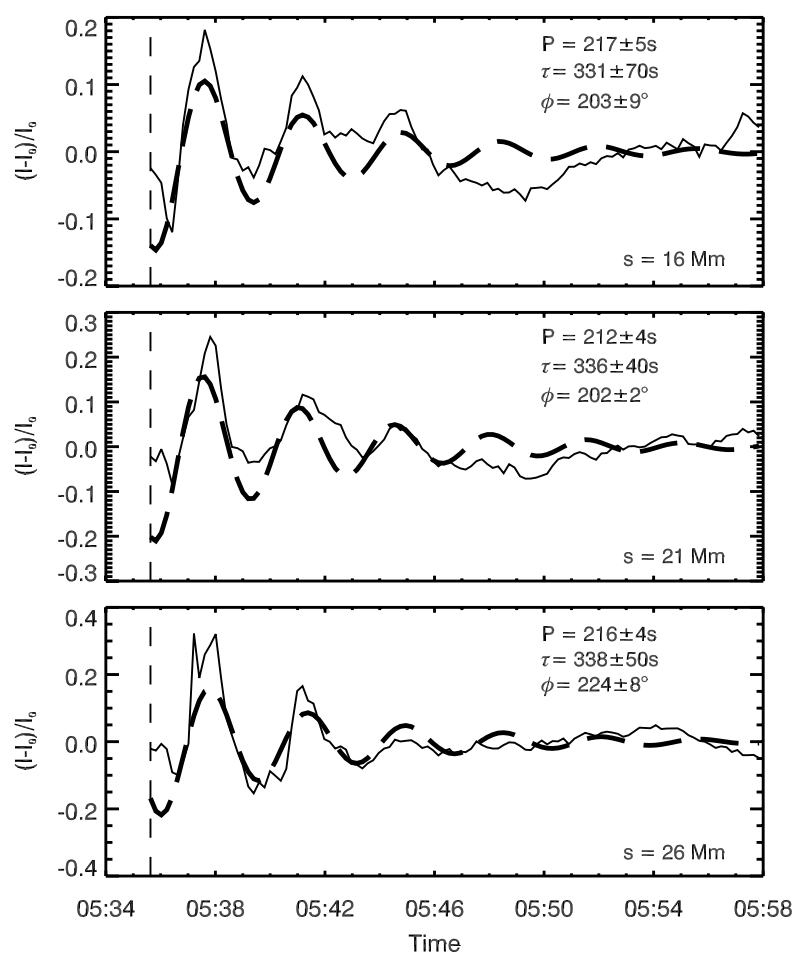

Fig. 6. Intensity time series analysis for loop 2 in Event 1 . The solid line shows the intensity time series which has been fitted with a damped cosine function (thick dashed line). The background subtracted intensity amplitude has been normalised by the background. Top: $s=16 \mathrm{Mm}$. Center: $s=21 \mathrm{Mm}$. Bottom: $s=26 \mathrm{Mm}$. The errors on the fitting parameters are the errors given by the fitting technique. The vertical dashed line indicates the reference time of 05:35:59 UT.

variations that are less reliably observed due to the contrast with the background plasma but for which an extracted time series can be fitted with a damped cosine function. For all except loop 1 in Event 2 the intensity and displacement oscillations are observed to be in phase or in antiphase. As the choice of direction of the cut across the loop is arbitrary, this means that the two types of oscillation are in phase. The phases have been corrected to the same reference times and the errors have been calculated in the same way as the time series analysis. The results show intensity periods that are similar to the displacement time periods except for loop 1 in Event 3. Figure 7 shows the intensity time series plots for loop 1 which shows that the period significantly changes along the loop. This may indicate that the intensity variations are resulting from a line of sight effect caused by loop paths crossing. Except for loop 1 of Event 3, the periods of the displacement and the intensity variations match within $2 \sigma$.

\subsection{Simulations of the intensity variations from the horizontally polarised mode}

With an estimate of the 3D loop geometry from the AIA-EUVI comparison, the intensity variations observed in loop 2, Event 1 shown in Fig. 6 are investigated further. The modelled loop is given a volume and a density contrast of 10 with the background and the loop radius is assumed to be $1 \mathrm{Mm}$. To simulate the motion the loop is perturbed as

$\boldsymbol{r}_{\text {loop }}=\boldsymbol{r}_{\text {loop }, 0}+\xi_{0} \cos (\phi) \sin \left(\frac{n \pi}{L} s\right) \boldsymbol{e}_{\perp}$

where $\boldsymbol{r}_{\text {loop }, 0}$ is the loop position at rest, $\xi_{0}$ is the amplitude (estimated to be $5.7 \mathrm{Mm}$ ), $\phi$ is the phase, $n$ indicates the harmonic, $s$ 
R. S. White and E. Verwichte: Transverse coronal loop oscillations seen in unprecedented detail by AIA/SDO
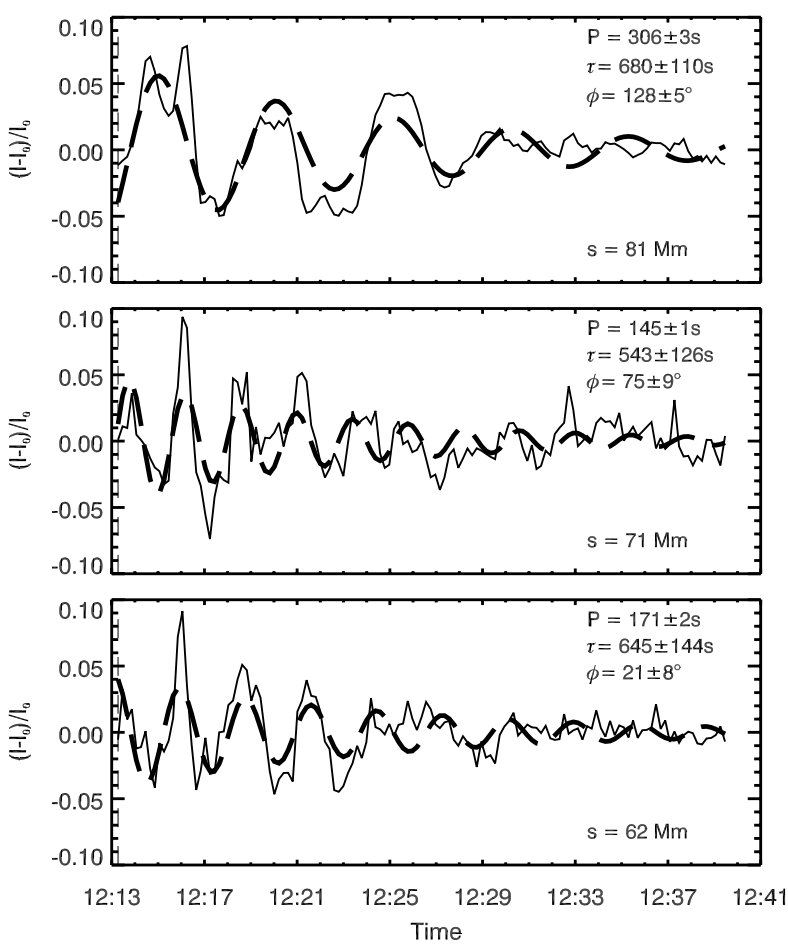

Fig. 7. Intensity time series analysis for loop 1 in Event 3 showing the variation of the period with the loop distance coordinate $s$. Top: $s=$ $81 \mathrm{Mm}$. Center: $s=71 \mathrm{Mm}$. Bottom: $s=62 \mathrm{Mm}$. The errors on the fitting parameters are the errors given by the fitting technique. The solid line shows the intensity time series which has been fitted with a damped cosine function (thick dashed line). The background subtracted intensity amplitude has been normalised by the background. The vertical dashed line indicates the reference time of 12:13:36 UT.

is the loop distance coordinate and $L$ is the loop length. The unit vector $\boldsymbol{e}_{\perp}$ is defined as $\boldsymbol{e}_{\perp}=-\boldsymbol{e}_{n} \sin (\theta)+\left(\boldsymbol{e}_{b} \times \boldsymbol{e}_{n}\right) \cos (\theta)$ where $\boldsymbol{e}_{n}$ is the photospheric normal unit vector and $\boldsymbol{e}_{b}$ is the unit vector parallel to the loop footpoints. A movie of the loop motion can be constructed in $3 \mathrm{D}$ and projected onto the image plane where the loop is uniformly filled and then analysed in the same way as the observational data set.

Figure 8 shows a difference image for the modelled horizontally polarised loop and a difference image from two AIA images. This and visual inspection of the AIA movie shows that the oscillation is horizontally polarised. (Therefore, the simulations are focussed on this mode rather than the vertically polarised mode). The pattern on the bottom leg of the simulated loop is matched by the difference image of the observed data set. Figure 9 shows the time distance images taken of the simulation data set. The three central figures correspond to the same position along the loop as for the cuts in Fig. 2. Figure 10 shows the automatically determined displacement time series of the simulations. As expected they show that as the distance along the loop increases towards the apex the displacement amplitude also increases. Figure 11 shows the corresponding intensity time series.

Here, clear intensity variations are observed, which can also be seen in the time distance images. In the simulations the intensity variations can only be due to variations in the line-of-sight column depth. The amplitude of the intensity variation at each location along the loop depends on the amount of displacement but also on the angle of the local loop axis unit vector and the line-of-sight. This explains what we observe. First, comparing Figs. 10 and 11 shows that towards the loop apex $(s \geq 21 \mathrm{Mm})$ the intensity amplitude indeed increases with the displacement.
Table 3. Seismology parameters.

\begin{tabular}{lccc}
\hline \hline Event & Loop & $\begin{array}{c}\text { Minimum } V_{\mathrm{A} 0} \\
\left(\mathrm{~km} \mathrm{~s}^{-1}\right)\end{array}$ & $\begin{array}{c}B \\
(\mathrm{G})\end{array}$ \\
\hline 1 & 1 & $761 \pm 155$ & $3.9-5.6$ \\
& 2 & $730 \pm 75$ & $3.8-5.3$ \\
& 3 & $876 \pm 95$ & $4.5-6.4$ \\
& 4 & $740 \pm 118$ & $3.8-5.4$ \\
\hline 2 & 1 & $1077 \pm 108$ & $5.6-7.9$ \\
& 2 & $887 \pm 116$ & $4.6-6.5$ \\
\hline 3 & 1 & $1861 \pm 256$ & $9.6-13.6$ \\
& 2 & $1326 \pm 175$ & $6.8-9.7$ \\
& 3 & $2524 \pm 303$ & $13.0-18.4$ \\
& 4 & $1955 \pm 199$ & $10.1-14.3$ \\
& 5 & $1812 \pm 230$ & $9.4-13.2$ \\
\hline
\end{tabular}

The exact match of the ratio of intensity and displacement amplitudes between observation and simulation depends on the size and structure of the loop cross-section (Verwichte et al. 2009). Towards the nearest footpoint $(s<21 \mathrm{Mm})$, the simulation shows an increase in intensity variations. There, the loop axes is oriented near the line-of-sight so that we look almost down onto the footpoint. Therefore, the integrated intensity there has an increased sensitivity to displacements that changes the orientation of the loop axis.

\section{Application of coronal seismology}

For the MHD kink mode in the long wavelength and zero plasma- $\beta$ limit, the phase speed is equal to the kink speed, $C_{\mathrm{k}}$, which can be written as $C_{\mathrm{k}}=\left(2 /\left(1+\rho_{\mathrm{e}} / \rho_{0}\right)\right)^{1 / 2} V_{\mathrm{A} 0}$ (Edwin $\&$ Roberts 1983) where $\rho_{\mathrm{e}}$ and $\rho_{0}$ are the external and internal densities and $V_{\mathrm{A} 0}$ is the internal Alfvén speed. Assuming that the density contrast $\rho_{\mathrm{e}} / \rho_{0}$ has extremes of 0 and 1 then the range of the internal Alfvén speed is given by the inequality $V_{\mathrm{A} 0} \leq V_{\mathrm{ph}} \leq \sqrt{2} V_{\mathrm{A} 0}$ (Nakariakov \& Ofman 2001). Following this, the range of the magnetic field can be written as

$\frac{V_{\mathrm{ph}}}{\sqrt{2}} \sqrt{\mu_{0} \tilde{\mu} m_{\mathrm{p}} n_{\mathrm{e}}} \leq B \leq V_{\mathrm{ph}} \sqrt{\mu_{0} \tilde{\mu} m_{\mathrm{p}} n_{\mathrm{e}}}$

where $\mu_{0}$ is the permeability, $\tilde{\mu}$ is the effective particle mass with respect to the proton mass $\left(m_{\mathrm{p}}\right)$ and has a value of 1.27 in the solar corona. The electron number density, $n_{\mathrm{e}}$ of the loops has an assumed value of $10^{15} \mathrm{~m}^{-3}$. If we assume the oscillation is damped by resonant mode conversion then the lower limit of the phase speed can be higher (Goossens et al. 2008). Table 3 shows the minimum internal Alfvén speed and the range of the magnetic field calculated from the analysis of the kink oscillations of the eleven coronal loops studied. The magnetic field estimates display a narrow range that are consistent with the range of 4 to $30 \mathrm{G}$ predicted by Nakariakov \& Ofman (2001). The uncertainty in the magnetic field values is calculated by $\delta B=B\left(\delta V_{\mathrm{ph}} / V_{\mathrm{ph}}\right)$. This gives an uncertainty of less than $21 \%$ for all values of the magnetic field. One of the main challenges for determining the magnetic field is estimating the density contrast between the loops and the background corona. This is especially challenging for on limb events where the column depth is larger than for on disk events. The largest magnetic field strengths are observed for loop 3 in Event 3. This could be a result of this loop being less dense. 

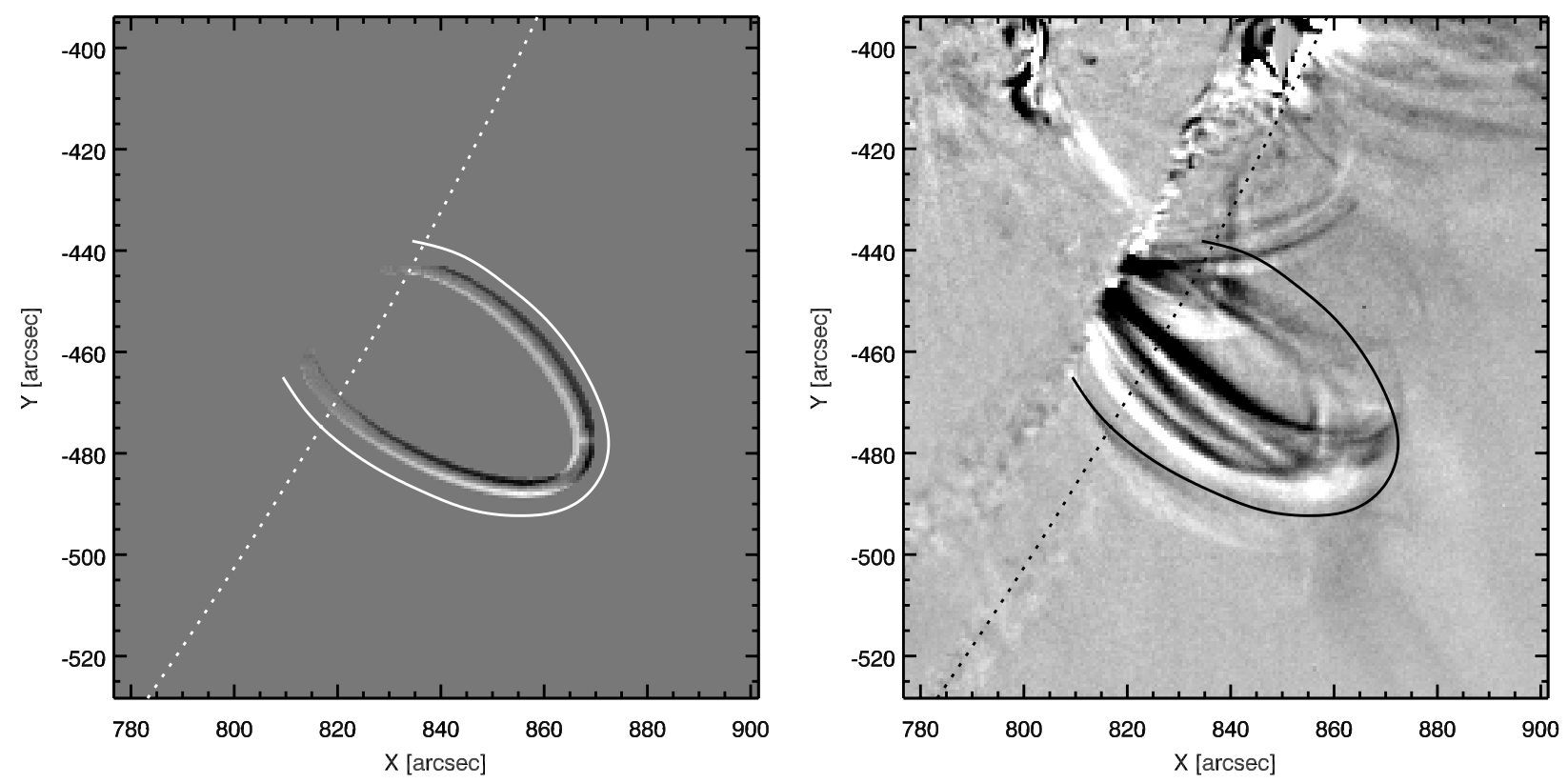

Fig. 8. Left: simulated difference image for the modelled horizontally polarised loop 2 from Event 1 . Right: difference image of Event 1 costructured by subtracting the image taken at 5:40:23 from the one at 5:38:23 UT. The dotted line indicates the solar limb.
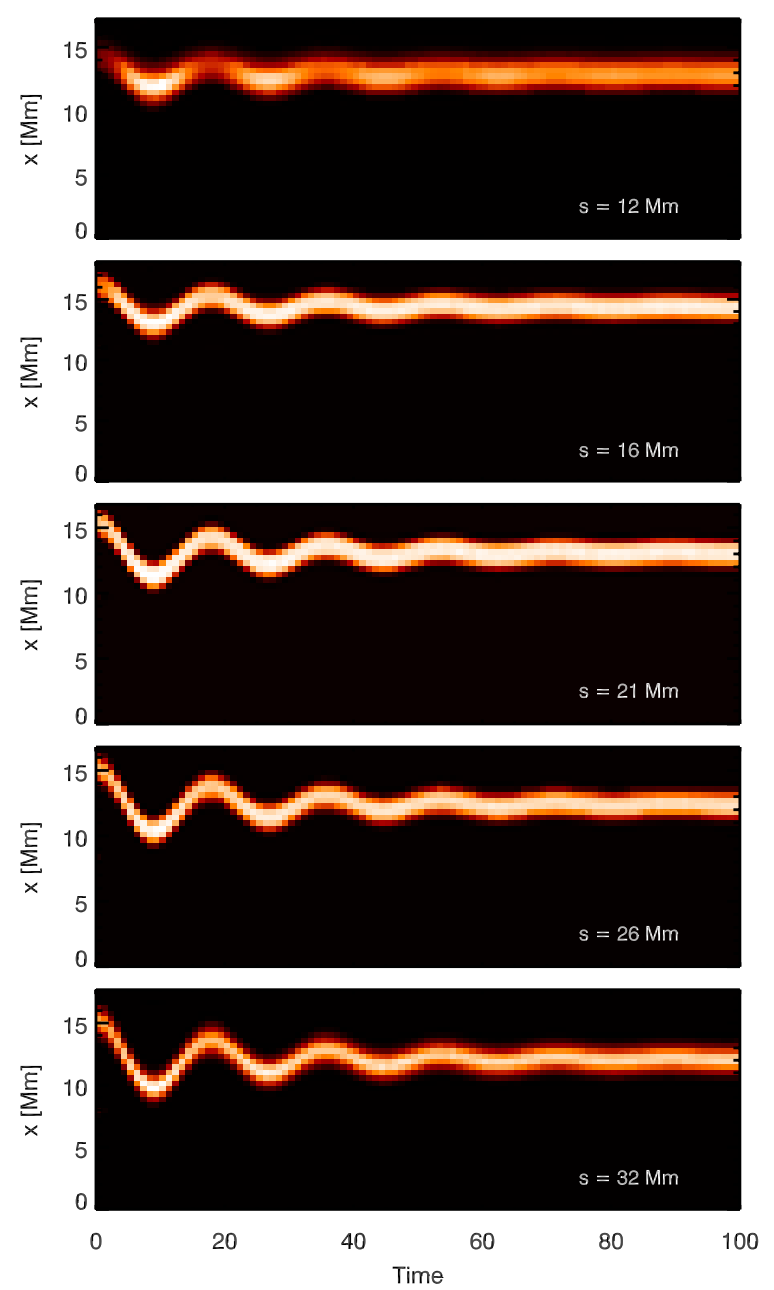

Fig. 9. Time distance images obtained from five cuts used to analyse the horizontally polarised simulations of loop 2 from Event 1 . The $s$ distance along the loop corresponds to the position of the cuts relative to the observed loop in the AIA image.
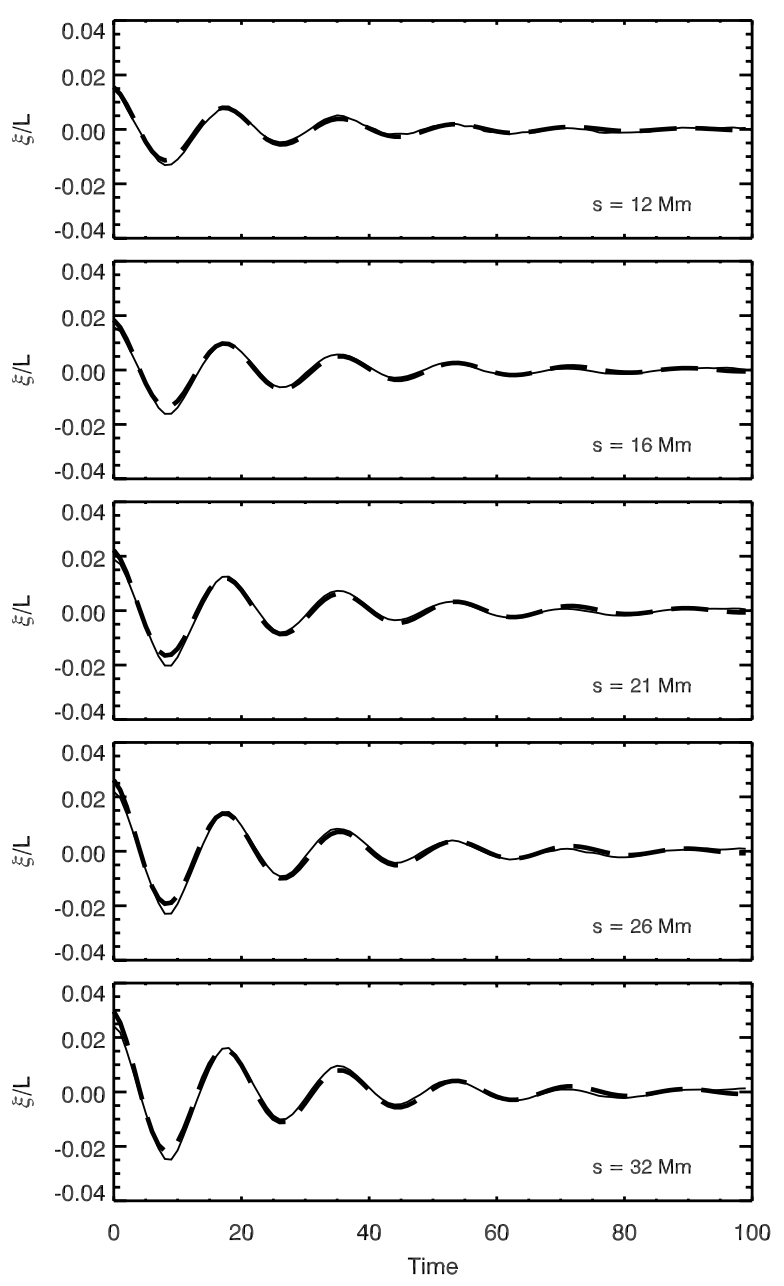

Fig. 10. Automatically determined time series (solid line) for the cuts displayed in Fig. 9 fitted with a damped cosine function (thick dashed line). The displacement amplitude has been normalised by the loop length. The $s$ distance along the loop corresponds to the position of the cuts relative to the observed loop in the AIA image. 
R. S. White and E. Verwichte: Transverse coronal loop oscillations seen in unprecedented detail by AIA/SDO
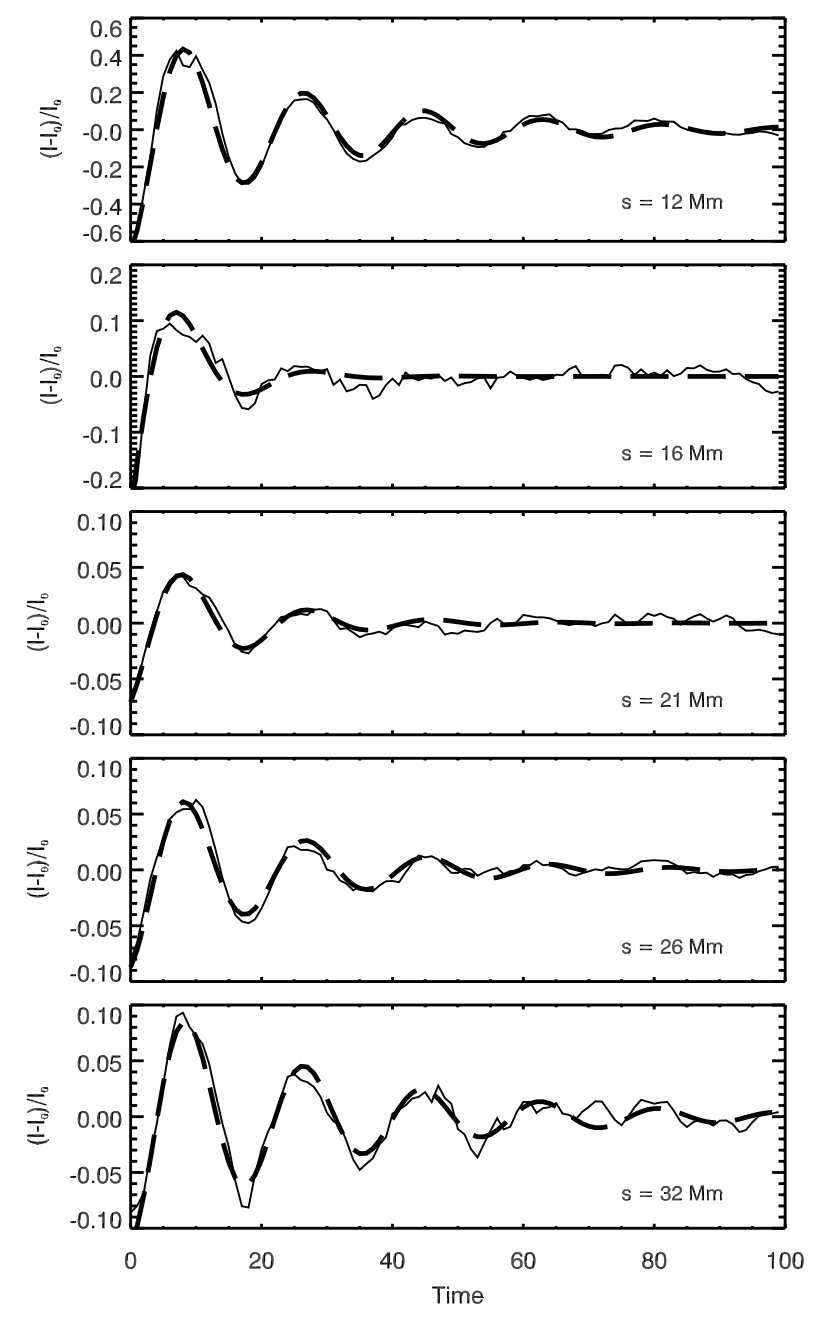

Fig. 11. Intensity time series analysis of the simulations of loop 2 from Event 1 assuming a horizontally polarised mode. The intensity time series (solid line) has been fitted with a damped cosine function (thick dashed line). The background subtracted intensity amplitude has been normalised by the background. The $s$ distance along the loop corresponds to the position of the cuts relative to the observed loop in the AIA image.

\section{Conclusions}

In this study the detailed analysis of eleven coronal loops observed in three events has been performed. The displacement time series analysis revealed periods between 1.7 and $10 \mathrm{~min}$ which are consistent with previous studies. Spatial observations of periods below 2 min have not been reported before as their detection requires instruments with short cadence times such as AIA/SDO. This study helps to build up a statistical picture of the behaviour of transverse loop oscillations which, along with coronal seismology provide a vital tool for coronal plasma diagnostics. The application of coronal seismology techniques of the MHD kink mode reveals magnetic field strengths in the range of 3 to $19 \mathrm{G}$ which are consistent with expectations. Narrowing down the range of the density contrast would provide a more accurate value for the magnetic field however the main contribution to the uncertainty in most cases is the large error on the loop length. Scaling relationships between the damping time and the period have been derived for the damping mechanisms of resonant absorption and phase mixing. Figure 12 shows a comparison between the results from the three events studied in this paper with previous studies of coronal loop oscillations and one prominence oscillation. Not only do the results from this study increase the number of reported events by about a third but they also extend the range to shorter periods and damping times than have previously been observed. Figure 12 gives a relationship between the damping time and period of $\tau=P^{0.98 \pm 0.09}$. Such a scaling can be reproduced by a resonant absorption damping mechanism, however this scaling should not be directly compared to the theoretical scaling because detailed information on loop properties is unavailable (Arregui et al. 2008). Directly comparing the damping time and periods of several events has been discussed by Hershaw et al. (2011). It was noted that the loops are not necessarily directly comparable because of the dependence on the damping time of the loop width and the thickness of the inhomogeneous layer which varies between loops. Nevertheless it shows that there is a relationship between the period and damping time of the loops. It is also noted that the damping times for Verwichte et al. (2004) may be biased towards longer damping times due to being semi driven by a prominence eruption close by. The observed periods and damping times presented in this paper are to be the subject of a further study where the technique of Bayesian seismology (Arregui \& Asensio Ramos 2011) is to be applied. Here the measurement errors are taken into account when inferring the range of coronal parameters such as the density contrast and Alfvén travel time.

There have been recent reports of intensity variations in transverse loop oscillations which are also observed in this study. The intensity oscillations are found to have similar periods to the displacement time oscillations except for loop 1 in Event 3. In this case the period was observed to vary along the loop with intensity periods differing from the time series periods.

From the comparison between EUVI and AIA data including the simulations of loop 2 in Event 1, and the fact that the displacement and intensity amplitudes are observed to increase with distance along the loop towards the loop apex (see Figs. 3, 6), it is expected that the intensity oscillations are due to line of sight effects of the horizontally polarised kink mode (Cooper et al. 2003; Verwichte et al. 2009, 2010). Other mechanisms such as non linear (Terradas \& Ofman 2004) and linear coupling effects (Terradas et al. 2011) which can cause intensity variations would not be expected to cause the variations here. Also, the strength of the linear coupling depends on the plasma- $\beta$. In active region loops with lengths of $100-200 \mathrm{Mm}$ the plasma- $\beta$ is expected to be small. For linear coupling the wavelength of the slow magnetoacoustic mode $\lambda_{\mathrm{s}}$ would approximately relate to the wavelength of the kink mode $\lambda_{\mathrm{k}}$ under coronal conditions as $\lambda_{\mathrm{s}} \cong\left(C_{\mathrm{s}} / C_{\mathrm{k}}\right) \lambda_{\mathrm{k}} \cong 0.15 \lambda_{\mathrm{k}}=0.3 L$. We have not observed variations in the intensity over such short distances along the loops. For the case of non linear coupling, the period of the kink mode $P_{\mathrm{k}}$ would approximately relate to the period of the slow mode $P_{\mathrm{s}}$ as $P_{\mathrm{k}} / P_{\mathrm{s}} \cong 2 C_{\mathrm{s}} / C_{\mathrm{k}}$ which under coronal conditions is expected to be much less than one. Observed intensity variations have periods that are similar to the displacement periods which would rule out non linear coupling for these cases.

The variations in intensity of loop 1 in Event 3 is an intriguing case as these are not easily explained by wave coupling or line of sight variations of the oscillating loop. Another potential explanation could be in terms of independently running slow waves. However, these would have short wavelengths and we would have observed phase shifts between different cuts, which we do not. It is possible that we see periods in intensity variations of approximately half the displacement periods if the variations were due to the loop periodically overlapping another structure. During each oscillation cycle we would then see two intensity 


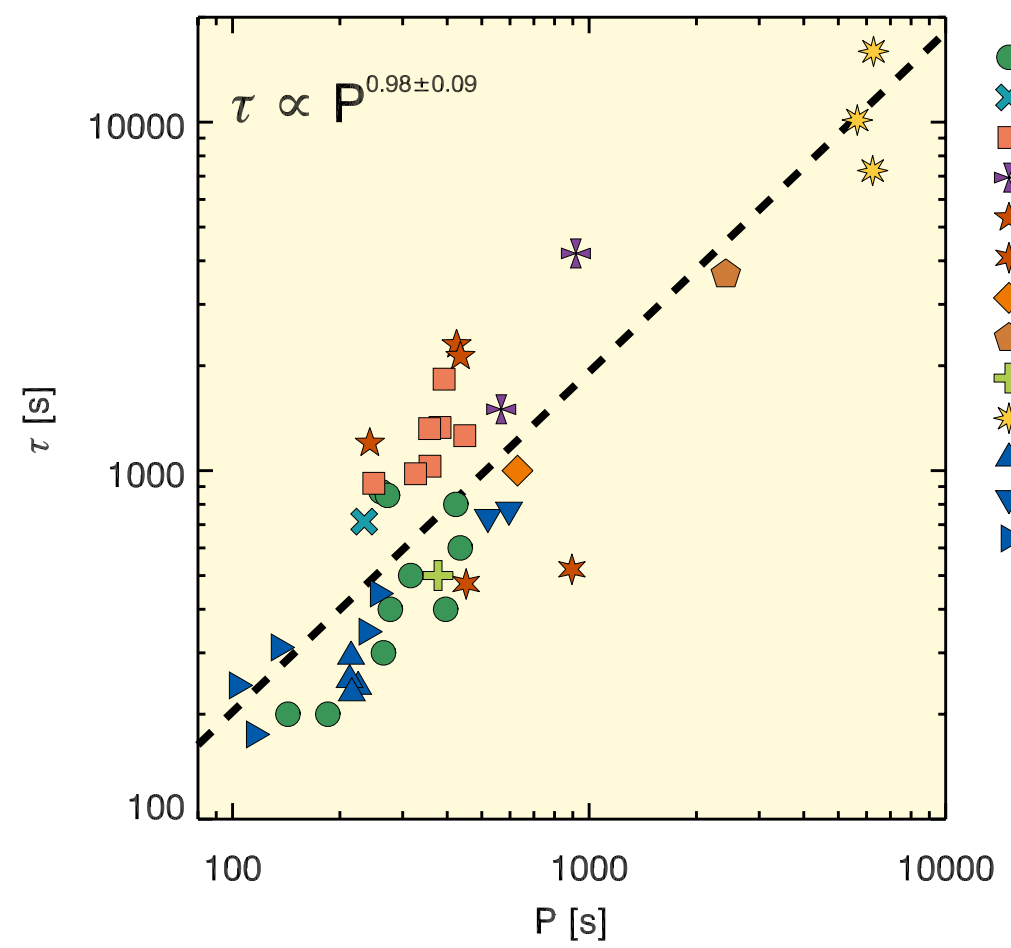

Aschwanden et al. (2002)
Wang \& Solanki (2004)
Verwichte et al. (2004)
Hori et al. (2005,2007)
Van Doorsselaere et al. (2007)
De Moortel \& Brady (2007), Van Doorsselaere et al. (2009)
Verwichte et al. (2009)
Mrozichte et al. (2010)
$\square$ Event 2
Event 3

Fig. 12. A comparison of the periods and damping times of Events 1, 2 and 3 with previous studies of transverse coronal loop oscillations and one prominence oscillation.

enhancements. However, for this observed loop the poor intensity contrast does not allow us to make a definite conclusion.

\section{References}

Arregui, I., Andries, J., Van Doorsselaere, T., Goossens, M., \& Poedts, S. 2007, A\&A, 463, 333

Arregui, I., \& Asensio Ramos, A. 2011, ApJ, 740, 44

Arregui, I., Ballester, J. L., \& Goossens, M. 2008, ApJ, 676, L77

Aschwanden, M. J., \& Schrijver, C. J. 2011, ApJ, 736, 102

Aschwanden, M. J., \& Terradas, J. 2008, ApJ, 686, L127

Aschwanden, M. J., Fletcher, L., Schrijver, C. J., \& Alexander, D. 1999, ApJ, 520,880

Aschwanden, M. J., de Pontieu, B., Schrijver, C. J., \& Title, A. M. 2002, Sol. Phys., 206, 99

Carcedo, L., Brown, D. S., Hood, A. W., Neukirch, T., \& Wiegelmann, T. 2003, Sol. Phys., 218, 29

Cheng, X., Zhang, J., Liu, Y., \& Ding, M. D. 2011, ApJ, 732, L25+

Cooper, F. C., Nakariakov, V. M., \& Tsiklauri, D. 2003, A\&A, 397, 765

De Moortel, I., \& Brady, C. S. 2007, ApJ, 664, 1210

Delouille, V., de Patoul, J., Hochedez, J. F., Jacques, L., \& Antoine, J. P. 2005, Sol. Phys., 228, 301

Edwin, P. M., \& Roberts, B. 1983, Sol. Phys., 88, 179

Foullon, C., Verwichte, E., Nakariakov, V. M., Nykyri, K., \& Farrugia, C. J. 2011, ApJ, 729, L8

Goossens, M., Andries, J., \& Aschwanden, M. J. 2002, A\&A, 394, L39

Goossens, M., Arregui, I., Ballester, J. L., \& Wang, T. J. 2008, A\&A, 484, 851

Goossens, M., Terradas, J., Andries, J., Arregui, I., \& Ballester, J. L. 2009, A\&A, 503,213

Hershaw, J., Foullon, C., Nakariakov, V. M., \& Verwichte, E. 2011, A\&A, 531, A53

Heyvaerts, J., \& Priest, E. R. 1983, A\&A, 117, 220

Hollweg, J. V., \& Yang, G. 1988, J. Geophys. Res., 93, 5423

Hori, K., Ichimoto, K., Sakurai, T., Sano, I., \& Nishino, Y. 2005, ApJ, 618, 1001
Hori, K., Ichimoto, K., \& Sakurai, T. 2007, in New Solar Physics with Solar-B Mission, ed. K. Shibata, S. Nagata, \& T. Sakurai, ASP Conf. Ser., 369, 213 Howard, R. A., Moses, J. D., Vourlidas, A., et al. 2008, Space Sci. Rev., 136, 67 Lemen, J. R., Title, A. M., Akin, D. J., et al. 2011, Sol. Phys., 115

Markwardt, C. B. 2009, in Astronomical Data Analysis Software and Systems XVIII, ed. D. A. Bohlender, D. Durand, \& P. Dowler, ASP Conf. Ser., 411, 251

McIntosh, S. W., de Pontieu, B., Carlsson, M., et al. 2011, Nature, 475, 477

Morton, R. J., \& Erdélyi, R. 2009, ApJ, 707, 750

Mrozek, T. 2011, Sol. Phys., 270, 191

Nakariakov, V. M., \& Ofman, L. 2001, A\&A, 372, L53

Nakariakov, V. M., \& Verwichte, E. 2005, Liv. Rev. Sol. Phys., 2, 3

Nakariakov, V. M., Ofman, L., Deluca, E. E., Roberts, B., \& Davila, J. M. 1999, Science, 285, 862

Ofman, L., \& Aschwanden, M. J. 2002, ApJ, 576, L153

Reeves, K. K., \& Golub, L. 2011, ApJ, 727, L52

Roberts, B. 2000, Sol. Phys., 193, 139

Ruderman, M. S., \& Roberts, B. 2002, ApJ, 577, 475

Terradas, J., \& Ofman, L. 2004, ApJ, 610, 523

Terradas, J., Andries, J., \& Verwichte, E. 2011, A\&A, 527, A132

Tomczyk, S., McIntosh, S. W., Keil, S. L., et al. 2007, Science, 317, 1192

Van Doorsselaere, T., Nakariakov, V. M., \& Verwichte, E. 2007, A\&A, 473, 959

Van Doorsselaere, T., Nakariakov, V. M., \& Verwichte, E. 2008a, ApJ, 676, L73

Van Doorsselaere, T., Nakariakov, V. M., Young, P. R., \& Verwichte, E. 2008b, A\&A, 487, L17

Van Doorsselaere, T., Birtill, D. C. C., \& Evans, G. R. 2009, A\&A, 508, 1485

Verwichte, E., Nakariakov, V. M., Ofman, L., \& Deluca, E. E. 2004, Sol. Phys., 223, 77

Verwichte, E., Foullon, C., \& Nakariakov, V. M. 2006, A\&A, 452, 615

Verwichte, E., Aschwanden, M. J., Van Doorsselaere, T., Foullon, C., \& Nakariakov, V. M. 2009, ApJ, 698, 397

Verwichte, E., Foullon, C., \& Van Doorsselaere, T. 2010, ApJ, 717, 458

Wang, T. J., \& Solanki, S. K. 2004, A\&A, 421, L33

Witkin, A. 1983, in Proc. Int. Joint Conf. Artificial Intell., Espoo, Finland 